\title{
HÖLDER CONTINUITY OF DEGENERATE $p$-HARMONIC FUNCTIONS
}

\author{
Flavia Giannetti and Antonia Passarelli di Napoli \\ Università di Napoli "Federico II", Dipartimento di Matematica e Applicazioni "R. Caccioppoli" \\ via Cintia - 80126 Napoli, Italia; giannett@unina.it \\ Università di Napoli "Federico II", Dipartimento di Matematica e Applicazioni "R. Caccioppoli" \\ via Cintia - 80126 Napoli, Italia; antonia.passarelli@unina.it
}

\begin{abstract}
We prove a partial Hölder continuity result for finite energy solutions of degenerate elliptic equations. The function that measures the degeneracy of the problem is assumed to belong to a suitable Sobolev class. Moreover, we prove an analogous result for infinite energy solutions provided their gradients have a suitable degree of integrability.
\end{abstract}

\section{Introduction}

Let us consider the equation

$$
\operatorname{div} A(x, D u)=0
$$

in a bounded domain $\Omega$ of $\mathbf{R}^{n}$. We suppose that $A: \Omega \times \mathbf{R}^{n} \rightarrow \mathbf{R}^{n}$ satisfies the following growth and ellipticity conditions

$$
\begin{aligned}
|A(x, \xi)| & \leq k(x)|\xi|^{p-1}, \\
\langle A(x, \xi), \xi\rangle & \geq \frac{1}{k(x)}|\xi|^{p}
\end{aligned}
$$

for almost every $x \in \Omega$, all $\xi \in \mathbf{R}^{n}$ and an exponent $1<p \leq n$.

We shall say that $u \in W_{\text {loc }}^{1,1}(\Omega)$ is a solution of the equation (1.1) if $A(x, D u)$ is locally integrable in $\Omega$ and $u$ satisfies the equation in the sense of distributions, that is,

$$
\int_{\Omega}\langle A(x, D u), D \varphi\rangle d x=0
$$

for every $\varphi \in C_{0}^{\infty}(\Omega)$. A function $u$ will be called a locally finite energy solution if, in addition, $\langle A(x, D u), D u\rangle$ is locally integrable in $\Omega$.

If $k$ is bounded, the equation is uniformly elliptic and finite energy solutions are those belonging to the class $W_{\text {loc }}^{1, p}(\Omega)$. The regularity of such solutions has been widely investigated and, for an exhaustive treatment of the argument, we refer the interested reader to $[10,11,12]$ and references therein.

Anyway, we recall that the study of the continuity properties of the solutions started, in the case $n=2$, with the pioneering papers by Morrey [19, 20] and, in higher dimensions, by De Giorgi [3] and Nash [18] and later on by Ladyzhenskaya and Ural'tseva [16].

doi:10.5186/aasfm.2014.3949

2010 Mathematics Subject Classification: Primary 35B65, 31B05.

Key words: Degenerate elliptic equations, Hölder's continuity. 
More recently, also the regularity of infinite energy solutions attracted the interest of many authors. It has been proven in $[14,17]$ that, if the gradient of a solution $u$ is assumed to be integrable with an exponent sufficiently close to the natural exponent $p$, then it is actually a finite energy solution.

In the case $k$ unbounded, the equation is degenerate elliptic and the study of the regularity of (finite and infinite energy) solutions started with the celebrated paper by Iwaniec and Sbordone ([15]) and, later on, developed in different directions by many authors (see for example $[1,8,13,21]$ ).

It is worth pointing out that the higher integrability of the gradient of the solutions has been usually established assuming that the function $k$, which measures the degree of degeneracy of the equation, is exponentially integrable, that is $\exp (\beta k) \in L^{1}(\Omega)$, for some $\beta>0$. In fact, in this case, finite energy solutions have a degree of integrability not too far from the natural one. More recently, the attention has been given also to the case $k$ subexponentially integrable, i.e., $\exp (P(k)) \in L^{1}(\Omega)$, for a suitable Orlicz function $P$ which is diverging at $\infty$ (see for example $[6,9]$ ).

As far as we know, the continuity of finite energy solutions under the assumption of exponentially integrable degeneracy, conjectured by De Giorgi in [4], is still open. More precisely

Conjecture 1.1. (De Giorgi) Let $u$ be a finite energy solution of the equation

$$
\operatorname{div}(A(x) \nabla u)=0 \quad \text { in } \Omega \subset \mathbf{R}^{n},
$$

where

$$
\frac{1}{k(x)}|\xi|^{2} \leq\langle A(x) \xi, \xi\rangle \leq|\xi|^{2}
$$

for almost every $x \in \Omega$ and all $\xi \in \mathbf{R}^{n}$. If

$$
\exp (k(x)) \in L^{1}(\Omega)
$$

then $u$ is continuous.

Many authors gave their contribution to prove this conjecture, but the exponentially integrability assumption on the degeneracy $k$ has been used together with stronger requirements. Among the others, we recall [22], where the continuity is obtained in the case $p=n=2$ and [24], where the author proves the continuity of local solutions to the linear non uniformly elliptic equation of the type

$$
\operatorname{div}(a(x) \nabla u)=0
$$

assuming that $a^{p} \in W^{1, n}, n \geq 3$, for some $p$ opportunely related to $n$ and $a(x) \geq$ $a_{0}>0$.

In this paper we establish the Hölder continuity of finite energy solutions of (1.1), under the assumption

$$
k \in W^{1, n}(\Omega) .
$$

In fact, our main result is the following

Theorem 1.2. Let $u$ be a finite energy solution of equation (1.1) and suppose that (1.2) and (1.3) hold for a function $k \in W^{1, n}(\Omega)$. Then there exists an open set $\Omega_{0} \subset \Omega$ with full measure such that

$$
u \in C^{0, \alpha}\left(\Omega_{0}\right)
$$

for every $\alpha \in(0,1)$. Moreover, $\operatorname{dim}_{H}\left(\Omega \backslash \Omega_{0}\right)=0$. 
In order to prove Theorem 1.2, the idea is to combine the isoperimetric type inequality, obtained in a more general setting in [7], with the methods of [9]. It is well known that the solutions of equation (1.1) are Hölder continuous if the operator $A(x, \xi)$ depends continuously on the variable $x$. Therefore, the assumption $k \in W^{1, n}$ could not appear adequate. Actually, we take advantage from this assumption since, via the isoperimetric type inequality and from the Sobolev imbedding theorem on spheres, we deal with $k$ as a continuous function on $\mathbf{R}^{n-1}$.

We pay the possible discontinuity of $k$ restricting ourselves to the set $\Omega_{0}$ where the mean value of $k$ is bounded (compare with [2]). We'd like to point out that, with respect to the above mentioned papers, here we are able to deal with non linear equations in any dimension $n$.

Since infinite energy solutions of the equation (1.1), whose degeneracy $k(x)$ is exponentially integrable, are actually finite energy solutions provided their degree of integrability is not too far from $L^{p}$, as a consequence of Theorem 1.2 and by virtue of the results in $[6,8]$, we have the following

Corollary 1.3. Let $u$ be a infinite energy solution of equation (1.1) and suppose that (1.2) and (1.3) hold for a function $k \in W^{1, n}(\Omega)$. Then there exist a positive exponent $\beta_{0}=\beta_{0}\left(p, n,\|k\|_{W^{1, n}(\Omega)}\right)$ and an open set $\Omega_{0} \subset \Omega$ with full measure such that if

$$
D u \in \frac{L^{p}}{\log ^{\beta} L}(\Omega)
$$

for every $\beta>\beta_{0}$, then

$$
u \in C^{0, \alpha}\left(\Omega_{0}\right)
$$

for every $\alpha \in(0,1)$. Moreover $\operatorname{dim}_{H}\left(\Omega \backslash \Omega_{0}\right)=0$.

We conclude by noting that the assumption $k \in W^{1, n}$ has been previously employed in [23] where an higher differentiability result for the gradient of the solution has been obtained. Therefore, the Hölder continuity can be deduced from the result of [23] by the use of Sobolev imbedding theorem, if $p$ and $n$ are opportunely related.

\section{Notation and preliminary results}

In this section we recall some standard definitions and collect several results that we will use to establish our main result. We indicate with $B_{R}(x) \equiv B(x, R)$ the open ball $\left\{y \in \mathbf{R}^{n}:|x-y|<R\right\}$ centered at the point $x \in \mathbf{R}^{n}$ and having radius $R>0$. We omit the center of the ball when no confusion arises. All the balls considered are concentric, unless differently specified.

If $u$ is an integrable function defined on $B_{R}(x)$, we indicate with

$$
u_{x, R}=f_{B_{R}(x)} u(x) d x=\frac{1}{\omega_{n} R^{n}} \int_{B_{R}(x)} u(x) d x
$$

the integral average of the function $u$ over the ball $B_{R}(x)$, where $\omega_{n}$ is the Lebesgue measure of $B_{1}(0)$. We also adopt the convention of writing $u_{R}$ instead of $u_{x, R}$ when the center of the ball is clear from the context.

Next result is a technical iteration lemma proven in [10, Lemma 2.1]. 
Lemma 2.1. Let $\bar{R}, a, b, \alpha, \beta$ be positive constants with $\beta<\alpha$. There exist $A, \bar{t}>0$ such that if $f$ is a nonnegative, nondecreasing function such that

$$
f(\rho) \leq a\left[\left(\frac{\rho}{R}\right)^{\alpha}+t\right] f(R)+b R^{\beta}
$$

for some $t<\bar{t}$ and for all $0<\rho<R \leq \bar{R}$, then we have

$$
f(\rho) \leq A\left(\frac{\rho}{R}\right)^{\beta}\left[f(R)+b R^{\beta}\right]
$$

for every $0<\rho<R \leq \bar{R}$.

We shall need the following Sobolev inequality on spheres as formulated by Gehring in [5].

Theorem 2.2. Let $k$ be a function in the Sobolev class $W_{\text {loc }}^{1, n \vartheta}(\Omega), \frac{n-1}{n}<\vartheta \leq 1$. Then

$$
\sup _{\partial B_{t}} k-\inf _{\partial B_{t}} k \leq c(n) t\left(f_{\partial B_{t}}|D k|^{n \vartheta} d \mathcal{H}^{n-1}\right)^{\frac{1}{n \vartheta}}
$$

for almost every radius $t \in(0, R)$, where $B_{R} \Subset \Omega$.

Next theorem relates the decay estimate for the gradient of a Sobolev function with its Hölder regularity properties ( see [12, Theorem 7.19])

Theorem 2.3. (Morrey's Lemma) Let $u \in W^{1,1}(\Omega)$ and suppose that there exist two positive constants $K, \alpha$, with $\alpha \leq 1$, such that

$$
\int_{B_{r}}|\nabla u| d x \leq K r^{n-1+\alpha}
$$

for all balls $B_{r} \subset \Omega$. Then $u \in C^{0, \alpha}(\Omega)$ and for every ball $B_{r} \subset \Omega$

$$
\operatorname{Osc}_{B_{r}} u \leq C K r^{\alpha} \text {. }
$$

Next result has been proven in [9, Lemma 3.5].

Lemma 2.4. Let $u$ be a finite energy solution of equation (1.1) and suppose that (1.2) and (1.3) hold for a function $k \in W^{1, n}(\Omega)$. Then we have

$$
\int_{\mathbf{R}^{n}}\langle A(x, D u), \eta D u\rangle d x \leq \int_{\mathbf{R}^{n}}|A(x, D u)||D \eta||u-c| d x
$$

for every $\eta \in C_{0}^{\infty}\left(\mathbf{R}^{n}\right)$ and for every constant $c$.

From previous lemma we deduce the following isoperimetric type inequality, already proven in a slightly different version in [9] (see also [7]). The proof is given for the reader's convenience.

Proposition 2.5. Let $u$ be a finite energy solution of equation (1.1) and suppose that (1.2) and (1.3) hold for a function $k \in W^{1, n}$. Then, for every $x_{0} \in \Omega$,

$$
\int_{B\left(x_{0}, r\right)} \frac{1}{k(x)}|D u|^{p} d x \leq c(n)\left(\int_{\partial B\left(x_{0}, r\right)} k(x)|D u|^{p-1}\left|u-u_{\partial B_{r}}\right| d \mathcal{H}^{n-1}\right)
$$

for almost every radius $0<r<\operatorname{dist}\left(x_{0}, \partial \Omega\right)$.

Proof. Let us set $B\left(x_{0}, t\right)=B_{t}$ and let us define on $B_{r}$ the function

$$
\eta_{\varepsilon}(x)=\min \left\{1, \frac{r-|x|}{\varepsilon}\right\} \text {. }
$$


Choosing $\eta(x)=\eta_{\varepsilon}(x)$ in $(2.2)$, we get

$$
\begin{aligned}
\int_{B_{r-\varepsilon}}\langle A(x, D u), D u\rangle d x \leq & \left|\int_{B_{r} \backslash B_{r-\varepsilon}}\langle A(x, D u), D u\rangle \frac{r-|x|}{\varepsilon} d x\right| \\
& +\int_{B_{r} \backslash B_{r-\varepsilon}}|A(x, D u)|\left|D\left(\frac{r-|x|}{\varepsilon}\right)\right||u-c| d x
\end{aligned}
$$

If we observe that $\frac{r-|x|}{\varepsilon}<1$ in $B_{r} \backslash B_{r-\varepsilon}$ and that the second integral in the right hand side can be written as

$$
\frac{1}{\varepsilon} \int_{r-\varepsilon}^{r}\left(\int_{\partial B_{\rho}}|A(x, D u)||D(r-|x|)||u-c| d \mathcal{H}^{n-1}\right) d \rho,
$$

taking the limit as $\varepsilon \rightarrow 0$, we obtain

$$
\int_{B_{r}}\langle A(x, D u), D u\rangle d x \leq \int_{\partial B_{r}}|A(x, D u)||u-c| d \mathcal{H}^{n-1} .
$$

Hence, by using assumption (1.2) in the right hand side and (1.3) in the left hand side of previous estimate and choosing $c=u_{\partial B_{r}}$, we obtain

$$
\int_{B_{r}} \frac{1}{k(x)}|D u|^{p} d x \leq c(n)\left(\int_{\partial B_{r}} k(x)|D u|^{p-1}\left|u-u_{\partial B_{r}}\right| d \mathcal{H}^{n-1}\right)
$$

i.e., the conclusion.

\section{The proof of the main result}

This section is devoted to the proof of Theorem 1.2. The starting point will consist in a decay estimate for the energy integral. In order to shorten the notation, in what follows we shall denote by

$$
\mathcal{K}_{R}:=\left(\int_{B_{R}}|D k|^{n} d x\right)^{\frac{1}{n}} \quad \text { with } B_{R} \Subset \Omega .
$$

Theorem 3.1. Let $u$ be a finite energy solution of equation (1.1). Assume that (1.2) and (1.3) hold for a function $k \in W^{1, n}(\Omega)$. Let $B_{2 R}$ be a ball contained in the set

$$
\Omega_{0}=\left\{x \in \Omega: \limsup _{r \rightarrow 0} f_{B_{r}} k d x<+\infty\right\} .
$$

Then there exist a constant $\tilde{A}>0$ and a positive exponent $\beta=\beta\left(n, \mathcal{K}_{R}\right)$ such that

$$
\int_{B_{r}} \frac{1}{k}|D u|^{p} d x \leq \tilde{A}\left(\frac{r}{R}\right)^{\beta} \int_{B_{R}} \frac{1}{k}|D u|^{p} d x,
$$

whenever $0<r<R$.

Proof. For every $i \in \mathbf{N}$, let us consider the interval

$$
\Delta_{i}:=\left(\frac{R}{2^{i}}, \frac{R}{2^{(i-1)}}\right)
$$

and the annulus

$$
A_{i}:=B_{\frac{R}{2^{(i-1)}}} \backslash B_{\frac{R}{2^{i}}} .
$$


Let us define the sets $E_{i}=E_{i}^{1} \cap E_{i}^{2} \cap E_{i}^{3}$ with

$$
\begin{aligned}
& E_{i}^{1}:=\left\{t \in \Delta_{i}: \int_{\partial B_{t}}|D k|^{n} d \mathcal{H}^{n-1} \leq \frac{12}{\left|\Delta_{i}\right|} \int_{A_{i}}|D k|^{n} d x\right\}, \\
& E_{i}^{2}:=\left\{t \in \Delta_{i}: \int_{\partial B_{t}} k d \mathcal{H}^{n-1} \leq \frac{12}{\left|\Delta_{i}\right|} \int_{A_{i}} k d x\right\}, \\
& E_{i}^{3}:=\left\{t \in \Delta_{i}: \int_{\partial B_{t}}\left(\frac{1}{k(x)}|D u|^{p}\right)^{\frac{n-1}{n}} d \mathcal{H}^{n-1} \leq \frac{12}{\left|\Delta_{i}\right|} \int_{A_{i}}\left(\frac{1}{k(x)}|D u|^{p}\right)^{\frac{n-1}{n}} d x\right\} .
\end{aligned}
$$

By Fubini's Theorem we have that

$$
\left|\mathcal{C}\left(E_{i}^{1}\right)\right| \leq \frac{\left|\Delta_{i}\right|}{12}, \quad\left|\mathcal{C}\left(E_{i}^{2}\right)\right| \leq \frac{\left|\Delta_{i}\right|}{12} \quad \text { and } \quad\left|\mathcal{C}\left(E_{i}^{3}\right)\right| \leq \frac{\left|\Delta_{i}\right|}{12},
$$

and therefore

$$
\left|E_{i}\right| \geq \frac{\left|\Delta_{i}\right|}{4}>0
$$

Choosing $r \in E_{i}$ so that inequalities (2.3) and (2.1) hold, we get

$$
\begin{aligned}
\int_{B_{\frac{R}{2^{2}}}} \frac{1}{k(x)}|D u|^{p} d x \leq & \int_{B_{r}} \frac{1}{k(x)}|D u|^{p} d x \leq c(n) \int_{\partial B_{r}} k(x)|D u|^{p-1}\left|u-u_{\partial B_{r}}\right| d \mathcal{H}^{n-1} \\
\leq & c(n) \sup _{\partial B_{r}} k(x) \int_{\partial B_{r}}|D u|^{p-1}\left|u-u_{\partial B_{r}}\right| d \mathcal{H}^{n-1} \\
\leq & c(n) \sup _{\partial B_{r}} k(x)\left(\int_{\partial B_{r}}|D u|^{\frac{p(n-1)}{n}} d \mathcal{H}^{n-1}\right)^{\frac{p-1}{p} \frac{n}{n-1}} \\
& \cdot\left(\int_{\partial B_{r}}\left|u-u_{\partial B_{r}}\right|^{\frac{p(n-1)}{n-p}} d \mathcal{H}^{n-1}\right)^{\frac{n-p}{p(n-1)}} \\
\leq & c(n) \sup _{\partial B_{r}} k(x)\left(\int_{\partial B_{r}}|D u|^{\frac{p(n-1)}{n}} d \mathcal{H}^{n-1}\right)^{\frac{n}{n-1}},
\end{aligned}
$$

where, in the last line, we also used Sobolev-Poincaré inequality on spheres for the function $u$. From estimate (3.3) we deduce that

$$
\begin{aligned}
\int_{B_{\frac{R}{2^{2}}}} \frac{1}{k(x)}|D u|^{p} d x \leq & c(n) \sup _{\partial B_{r}} k(x)\left(\int_{\partial B_{r}}|D u|^{\frac{p(n-1)}{n}} d \mathcal{H}^{n-1}\right)^{\frac{n}{n-1}} \\
= & c(n) \sup _{\partial B_{r}} k(x)\left(\int_{\partial B_{r}} k^{\frac{n-1}{n}}\left(\frac{1}{k}|D u|^{p}\right)^{\frac{n-1}{n}} d \mathcal{H}^{n-1}\right)^{\frac{n}{n-1}} \\
\leq & c(n)\left(\sup _{\partial B_{r}} k(x)\right)^{2}\left(\int_{\partial B_{r}}\left(\frac{1}{k}|D u|^{p}\right)^{\frac{n-1}{n}} d \mathcal{H}^{n-1}\right)^{\frac{n}{n-1}} \\
\leq & c(n)\left(\sup _{\partial B_{r}} k(x)-\inf _{\partial B_{r}} k(x)\right)^{2}\left(\int_{\partial B_{r}}\left(\frac{1}{k}|D u|^{p}\right)^{\frac{n-1}{n}} d \mathcal{H}^{n-1}\right)^{\frac{n}{n-1}} \\
& +c(n)\left(\inf _{\partial B_{r}} k(x)\right)^{2}\left(\int_{\partial B_{r}}\left(\frac{1}{k}|D u|^{p}\right)^{\frac{n-1}{n}} d \mathcal{H}^{n-1}\right)^{\frac{n}{n-1}}
\end{aligned}
$$


Using Theorem 2.2 in (3.4), we get

$$
\begin{aligned}
\int_{B_{\frac{R}{2^{i}}}} \frac{1}{k(x)}|D u|^{p} d x \leq & c(n) r^{\frac{2}{n}}\left(\int_{\partial B_{r}}|D k|^{n} d \mathcal{H}^{n-1}\right)^{\frac{2}{n}}\left(\int_{\partial B_{r}}\left(\frac{1}{k}|D u|^{p}\right)^{\frac{n-1}{n}} d \mathcal{H}^{n-1}\right)^{\frac{n}{n-1}} \\
& +c(n)\left(f_{\partial B_{r}} k d \mathcal{H}^{n-1}\right)^{2}\left(\int_{\partial B_{r}}\left(\frac{1}{k}|D u|^{p}\right)^{\frac{n-1}{n}} d \mathcal{H}^{n-1}\right)^{\frac{n}{n-1}}
\end{aligned}
$$

where we used the obvious inequality

$$
\inf _{\partial B_{r}} k(x) \leq f_{\partial B_{r}} k(x) d \mathcal{H}^{n-1} .
$$

Since $r \in E_{i}$, from previous inequality we obtain

$$
\begin{aligned}
\int_{B_{\frac{R}{2^{i}}}} \frac{1}{k(x)}|D u|^{p} d x \leq & c(n)\left[r^{\frac{2}{n}}\left(\frac{1}{\left|\Delta_{i}\right|} \int_{A_{i}}|D k|^{n} d x\right)^{\frac{2}{n}}+\left(\frac{1}{r^{n-1}\left|\Delta_{i}\right|} \int_{A_{i}} k d x\right)^{2}\right] \\
& \cdot\left(\frac{1}{\left|\Delta_{i}\right|} \int_{A_{i}}\left(\frac{1}{k}|D u|^{p}\right)^{\frac{n-1}{n}} d x\right)^{\frac{n}{n-1}} .
\end{aligned}
$$

In order to shorten the notation, set

$$
\Gamma:=\left[r^{\frac{2}{n}}\left(\frac{1}{\left|\Delta_{i}\right|} \int_{A_{i}}|D k|^{n} d x\right)^{\frac{2}{n}}+\left(\frac{1}{r^{n-1}\left|\Delta_{i}\right|} \int_{A_{i}} k d x\right)^{2}\right]
$$

so that (3.5) can be written as

$$
\int_{B_{\frac{R}{2^{i}}}} \frac{1}{k(x)}|D u|^{p} d x \leq c(n) \Gamma\left(\frac{1}{\left|\Delta_{i}\right|} \int_{A_{i}}\left(\frac{1}{k}|D u|^{p}\right)^{\frac{n-1}{n}} d x\right)^{\frac{n}{n-1}} .
$$

By using Hölder's inequality and the fact that, since

$$
\left|A_{i}\right|=C(n) \frac{\left(2^{n}-1\right) R^{n}}{2^{n i}}, \quad\left|\Delta_{i}\right|=\frac{R}{2^{i}},
$$

we have

$$
\frac{\left|A_{i}\right|^{\frac{1}{n-1}}}{\left|\Delta_{i}\right|^{\frac{n}{n-1}}}=C(n)
$$

from (3.6), we deduce that

$$
\begin{aligned}
\int_{B_{\frac{R}{2^{i}}}} \frac{1}{k(x)}|D u|^{p} d x & \leq c(n) \Gamma\left(\frac{1}{\left|\Delta_{i}\right|}\right)^{\frac{n}{n-1}}\left|A_{i}\right|^{\frac{1}{n-1}} \int_{A_{i}} \frac{1}{k}|D u|^{p} d x \\
& \leq c(n) \Gamma \frac{R}{2^{i}} \frac{1}{\left|\Delta_{i}\right|} \int_{A_{i}} \frac{1}{k}|D u|^{p} d x
\end{aligned}
$$

In order to estimate $\Gamma$, we take into account that

$$
\left|\Delta_{i}\right|=\frac{R}{2^{i}} \text { and } \quad \frac{R}{2^{i}}<r<\frac{R}{2^{i-1}}
$$

and that, by virtue of the assumption $B_{2 R} \subset \Omega_{0}$, we may suppose the existence of a constant $L>1$ such that

$$
f_{B_{R}} k d x \leq L
$$


Hence, we have

$$
\begin{aligned}
\Gamma & \leq 2^{\frac{2}{n}}\left(\int_{A_{i}}|D k|^{n} d x\right)^{\frac{2}{n}}+2^{2 i n}\left(\frac{1}{R^{n}} \int_{B_{R}} k d x\right)^{2} \\
& \leq c(n, L)\left[\left(\int_{A_{i}}|D k|^{n} d x\right)^{\frac{2}{n}}+2^{2 i n}\right] .
\end{aligned}
$$

Inserting estimate (3.8) in (3.7), it follows

$$
\int_{B_{\frac{R}{2^{i}}}} \frac{1}{k(x)}|D u|^{p} d x \leq c(n, L) \frac{R 2^{-i}}{\left|\Delta_{i}\right|}\left[\left(\int_{A_{i}}|D k|^{n} d x\right)^{\frac{2}{n}}+2^{2 i n}\right] \int_{A_{i}} \frac{1}{k}|D u|^{p} d x
$$

Now, for $t \in \Delta_{i}$, we set

$$
v_{i}(t):=\int_{B_{\frac{R}{2^{i}}}} \frac{1}{k(x)}|D u|^{p} d x+c(n, L) \frac{t-R 2^{-i}}{\left|\Delta_{i}\right|} \int_{A_{i}} \frac{1}{k(x)}|D u|^{p} d x
$$

and

$$
v(t):=v_{1}(t) \chi_{\left[\frac{R}{2}, R\right]}(t)+\sum_{i=2}^{\infty} v_{i}(t) \chi_{\left[\frac{R}{2^{i}}, \frac{R}{2^{(i-1)}}\right)}(t) .
$$

Estimate (3.9) implies that

$$
v_{i}(t) \leq c(n, L) \frac{1}{\left|\Delta_{i}\right|}\left\{\frac{R}{2^{i}}\left[\left(\int_{A_{i}}|D k|^{n} d x\right)^{\frac{2}{n}}+2^{2 i n}\right]+t-\frac{R}{2^{i}}\right\} \int_{A_{i}} \frac{1}{k(x)}|D u|^{p} d x .
$$

Since

$$
v_{i}^{\prime}(t)=c(n, L) \frac{1}{\left|\Delta_{i}\right|} \int_{A_{i}} \frac{1}{k(x)}|D u|^{p} d x
$$

we obtain

$$
v_{i}(t) \leq\left\{\frac{R}{2^{i}}\left[\left(\int_{A_{i}}|D k|^{n} d x\right)^{\frac{2}{n}}+2^{2 i n}\right]+t-\frac{R}{2^{i}}\right\} v_{i}^{\prime}(t)
$$

for all $t \in \Delta_{i}$. Since $t-\frac{R}{2^{i}} \leq \frac{R}{2^{(i-1)}}-\frac{R}{2^{i}}=\frac{R}{2^{i}}$, from (3.10) we deduce

$$
\begin{aligned}
v_{i}(t) & \leq \frac{R}{2^{i}}\left[\left(\int_{A_{i}}|D k|^{n} d x\right)^{\frac{2}{n}}+2^{2 i n}+1\right] v_{i}^{\prime}(t) \\
& \leq \frac{2 R}{2^{i}}\left[\left(\int_{B_{R}}|D k|^{n} d x\right)^{\frac{2}{n}}+2^{2 i n}\right] v_{i}^{\prime}(t) .
\end{aligned}
$$

Using that $\frac{R}{2^{i}} \leq t \leq \frac{R}{2^{(i-1)}}$ in (3.11), it follows that

$$
v_{i}(t) \leq 2^{2 n+1} t\left[\mathcal{K}_{R}^{2}+\frac{R^{2 n}}{t^{2 n}}\right] v_{i}^{\prime}(t)
$$

and hence, summing on $i$ and observing that $v(t)$ is a piecewise affine function, we get

$$
v(t) \leq 2^{2 n+1} t\left[\mathcal{K}_{R}^{2}+\frac{R^{2 n}}{t^{2 n}}\right] v^{\prime}(t)
$$


for every $0<t<R$. Therefore

$$
\frac{v^{\prime}(t)}{v(t)} \geq \frac{1}{2^{2 n+1}} \frac{t^{2 n-1}}{\mathcal{K}_{R}^{2} t^{2 n}+R^{2 n}}
$$

Now, we easily obtain for every $\rho<R$

$$
\int_{\rho}^{R} \frac{v^{\prime}(t)}{v(t)} d t \geq \frac{1}{2^{2 n+1}} \int_{\rho}^{R} \frac{t^{2 n-1}}{\mathcal{K}_{R}^{2} t^{2 n}+R^{2 n}} d t=\frac{1}{n 2^{2 n+2} \mathcal{K}_{R}^{2}} \log \frac{\mathcal{K}_{R}^{2} R^{2 n}+R^{2 n}}{\mathcal{K}_{R}^{2} \rho^{2 n}+R^{2 n}}
$$

and therefore

$$
\log \frac{v(R)}{v(\rho)} \geq \frac{1}{n 2^{2 n+2} \mathcal{K}_{R}^{2}} \log \frac{\mathcal{K}_{R}^{2} R^{2 n}+R^{2 n}}{\mathcal{K}_{R}^{2} \rho^{2 n}+R^{2 n}}=\log \left(\frac{\mathcal{K}_{R}^{2} R^{2 n}+R^{2 n}}{\mathcal{K}_{R}^{2} \rho^{2 n}+R^{2 n}}\right)^{\alpha(n, R)},
$$

where we used the notation

$$
\alpha(n, R):=\frac{1}{n 2^{2 n+2} \mathcal{K}_{R}^{2}} .
$$

Inequality (3.13) yields

$$
v(\rho) \leq\left(\frac{\rho^{2 n} \mathcal{K}_{R}^{2}+R^{2 n}}{R^{2 n} \mathcal{K}_{R}^{2}+R^{2 n}}\right)^{\alpha(n, R)} v(R) \leq c(n, R)\left(\left(\frac{\rho}{R}\right)^{2 n \alpha(n, R)}+1\right) v(R),
$$

for every $0<\rho<R$. By Lemma 2.1 and estimate (3.14), we infer that there exists a positive constant $A$ such that

$$
v(\rho) \leq A\left(\frac{\rho}{R}\right)^{\beta} v(R)
$$

for every $\rho<R$ and for every

$$
\beta<2 n \alpha(n, R)=\frac{1}{2^{2 n+1} \mathcal{K}_{R}^{2}} .
$$

Since $\rho<R$, there exists $j \in \mathbf{N}$ such that $\rho \in\left[R 2^{-j}, R 2^{-j+1}\right)$. Then, by the definition of the function $v(t)$, from (3.15) it follows

$$
\int_{B_{\frac{R}{2 j}}} \frac{1}{k}|D u|^{p} d x+c(n, L) \frac{\rho-R 2^{-j}}{\left|\Delta_{j}\right|}\left[\int_{A_{j}} \frac{1}{k}|D u|^{p} d x\right] \leq A\left(\frac{\rho}{R}\right)^{\beta} \int_{B_{R}} \frac{1}{k}|D u|^{p} d x
$$

which obviously implies

$$
\int_{B_{\frac{R}{2^{j}}}} \frac{1}{k}|D u|^{p} d x \leq A\left(\frac{\rho}{R}\right)^{\beta} \int_{B_{R}} \frac{1}{k}|D u|^{p} d x .
$$

At this point, choosing $r=\frac{\rho}{2}<\frac{R}{2}$, we get

$$
\int_{B_{r}} \frac{1}{k}|D u|^{p} d x \leq \tilde{A}\left(\frac{r}{R}\right)^{\beta} \int_{B_{R}} \frac{1}{k}|D u|^{p} d x
$$

which concludes the proof.

Now, we are ready to embark in the core of the proof of our main result.

Proof of Theorem 1.2. Let us fix a ball $B_{2 R}$ contained in the set $\Omega_{0}$ defined at (3.1). The assumption $k \in W^{1, n}(\Omega)$ implies, through the Sobolev imbedding 
Theorem that $k \in L^{q}(\Omega)$, for every $q>1$. Hence, for every $0<r<\frac{R}{2}$, Hölder's inequality yields

$\int_{B_{r}}|D u| d x \leq\left(\int_{B_{r}} \frac{1}{k}|D u|^{p} d x\right)^{\frac{1}{p}}\left(\int_{B_{r}} k^{\frac{1}{p-1}}\right)^{\frac{p-1}{p}} \leq c\left(\|k\|_{W^{1, n}(\Omega)}\right)\left(\int_{B_{r}} \frac{1}{k}|D u|^{p} d x\right)^{\frac{1}{p}}$.

Therefore, by the assumption (1.3) and estimate (3.2), we obtain

$$
\int_{B_{r}}|D u| d x \leq C r^{\frac{\beta}{p}}
$$

In order to use Morrey's Lemma in Theorem 2.3, we need that $\beta$ satisfies

$$
0<\frac{\beta}{p}-n+1 \leq 1 \quad \Longleftrightarrow \quad(n-1) p<\beta \leq n p .
$$

Since $\beta<\frac{1}{2^{2 n+1} \mathcal{K}_{R}^{2}}$, such choice is possible if

$$
\mathcal{K}_{R}^{2}<\frac{1}{p 2^{2 n+1}(n-1)} .
$$

In order to obtain the Hölder continuity, it is sufficient to choose a radius $R<R_{0}$ such that

$$
\left(\int_{B_{R_{0}}}|D k|^{n} d x\right)^{\frac{2}{n}}<\frac{1}{p 2^{2 n+1}(n-1)},
$$

that is possible thanks to the absolute continuity of the integral.

Observe now that, for a function belonging to the Sobolev space $W^{1, p}(\Omega)$, with $1<p<n$, one has $\operatorname{dim}_{H}\left(\Omega \backslash \Omega_{0}\right) \leq n-p$, where $\Omega_{0}$ is the set defined at (3.1) (see for example Theorem 3.2 in [11]). Since $D k \in L^{n}(\Omega)$, we have $\operatorname{dim}_{H}\left(\Omega \backslash \Omega_{0}\right) \leq n-p$ for all $p<n$. Hence, taking the limit as $p$ goes to $n$, we have $\operatorname{dim}_{H}\left(\Omega \backslash \Omega_{0}\right)=0$.

We conclude with the

Proof of Corollary 1.3. It suffices to observe that, through the classical Moser Trudinger inequality [12], the function $k$ is exponentially integrable, i.e.,

$$
\int_{\Omega} \exp \left(\lambda k^{\frac{n}{n-1}}\right) \mathrm{d} x<+\infty
$$

for some constant $\lambda>0$ depending on the $W^{1, n}$-norm of $k$. By virtue of the results in $[6,8]$, we have that there exists a positive exponent $\beta_{0}=\beta_{0}\left(p, n,\|k\|_{W^{1, n}(\Omega)}\right)$ such that, if

$$
D u \in \frac{L^{p}}{\log ^{\beta} L}(\Omega)
$$

for every $\beta>\beta_{0}$, then $u$ is a finite energy solution. Once we have that $u$ is a finite energy solution, we may use Theorem 1.2 to conclude that there exists an open set $\Omega_{0} \subset \Omega$ with full measure such that $u \in C^{0, \alpha}\left(\Omega_{0}\right)$, for every $\alpha \in(0,1)$.

\section{References}

[1] Carozza, M., G. Moscariello, and A. Passarelli di Napoli: Regularity for $p$-harmonic equations with right hand side in Orlicz-Zygmund classes. - J. Differential Equations 242:2, 2007, 248-268.

[2] Cruz-Uribe, D., P. Di Gironimo, and C. Sbordone: On the continuity of solutions of degenerate elliptic equations. - J. Differential Equations 250, 2011, 2671-2686. 
[3] De Giorgi, E.: Sulla differenziabilitá e l' analiticitá delle estremali degli integrali multipli regolari. - Mem. Accad. Sci. Torino Cl. Sci. Fis. Mat. Natur. 3, 1957, 25-43.

[4] De Giorgi, E.: Congetture sulla continuitá delle soluzioni di equazioni lineari ellittiche autoaggiunte a coefficienti illimitati. - Unpublished, 1995.

[5] Gehring, F.: Rings and quasiconformal mapping in the space. - Trans. Amer. Math. Soc. 103, 1962, 353-393.

[6] Giannetti, F., L. Greco, and A. Passarelli di Napoli: Regularity of solutions of degenerate $A$-harmonic equations. - Nonlinear Anal. 73, 2010, 2651-2665.

[7] Giannetti, F., and A. Passarelli di Napoli: Isoperimetric type inequalities for differential forms on manifolds. - Indiana Univ. Math. J. 54:5, 2005, 1483-1497.

[8] Giannetti, F., and A. Passarelli di Napoli: On very weak solutions of degenerate equations. - NoDEA Nonlinear Differential Equations Appl. 14, 2007, 739-751.

[9] Giannetti, F., and A. Passarelli di Napoli: On the continuity of degenerate $n$-harmonic functions. - ESAIM Control Optim. Calc. Var. 18:3, 2012, 621-642.

[10] Giaquinta, M.: Multiple integrals in the calculus of variations and nonlinear elliptic systems. - Ann. of Math. Stud. 105, Princeton Univ. Press, 1983.

[11] Giusti, E.: Direct methods in the calculus of variations. - World Scientific, 2003.

[12] Gilbarg, D., and Trudinger, N. S.: Elliptic partial differential equations of second order. Second edition. - Grundlehren Math. Wiss. 224, Springer-Verlag, Berlin, 1983.

[13] Imaniec, T., L. Migliaccio, G. Moscariello, and A. Passarelli di Napoli: A priori estimates for nonlinear elliptic complexes. - Adv. Differentail Equations 8:5, 2003, 513-546

[14] Iwaniec, T., and C. Sbordone: Weak minima of variational integrals. - J. Reine Angew. Math. 454, 1994, 143-161.

[15] Iwaniec, T., and C. Sbordone: Quasiharmonic fields. - Ann. Inst. H. Poincaré Anal. Non Linéairse 18, 2001, 519-572.

[16] Ladyzhenskaya, O. A., and N. N. Ural'tseva: Linear and quasilinear elliptic equations. Academic Press, New York, 1968.

[17] Lewis, J.: On very weak solutions of certain elliptic systems. - Comm. Partial Differential Equations 18:9-10, 1993, 1515-1537.

[18] Nash, J.: Continuity of solutions of elliptic and parabolic equations. - Amer. J. Math. 80, $1958,931-954$.

[19] Morrey, C. B.: On the solutions of quasi-linear elliptic partial differential equations. - Trans. Amer. Math. Soc. 43, 1938, 126-166.

[20] Morrey, C. B.: Multiple integral problems in the calculus of variations and related topics. Univ. California Publ. Math. (N. S.) 1, 1943, 1-130.

[21] Moscariello, G.: On the integrability of finite energy solutions for $p$-harmonic equations. NoDEA Nonlinear Differential Equations Appl. 11, 2004, 393-406.

[22] Onninen, J., and X. Zhong: Continuity of solutions of linear degenerate elliptic equations. Ann. Sc. Norm. Super. Pisa Cl. Sci. (5) 6, 2007, 103-116.

[23] Passarelli di Napoli, A.: Higher differentiability of minimizers of variational integrals with Sobolev coefficients. - Adv. Calc. Var. 7:1, 2012, 59-89.

[24] Percivale, D.: Continuity of solutions of a class of linear non uniformly elliptic equations. Ric. Mat. 48:2, 1999, 249-258. 